\title{
Time-resolved serial synchrotron crystallography for the functional characterization of proteins
}

\author{
P Mehrabi ${ }^{1}$ \\ ${ }^{1}$ Max Planck Institute for the Structure and Dynamics of Matter, Hamburg \\ pedram.mehrabi@Mpsd.mpg.de
}

Functional characterization of proteins requires insights into the interplay between structure and dynamics as a function of time. Classical approaches to X-ray crystallography via populating intermediate states by mutation or chemical trapping only provide static time-averaged data sets. Considering this, serial time-resolved approaches are powerful tools to elucidate time-dependent structures. To this end we combined an established fixed target mount (chip), with a novel "Hit And REturn" (HARE) approach for data acquisition - enabling efficient data collection at microfocus beamlines. With the HARE algorithm a plethora of time delays are accessible from a few milliseconds to several minutes without the burden of an increase in data collection time. Using this approach, we mapped the catalytic cycle of a homodimeric enzyme, fluoroacetate dehalogenase, using a photo-caged substrate and initiating the reaction via laser excitation. A total of 18 time points was collected from 30 milliseconds to 30 seconds capturing key states involved in the reaction pathway. These include substrate binding and reorientation, covalentintermediate formation, location of the water nucleophile and product release. As well as interactions between the two active sites displayed in molecular breathing and a chain of water molecules modulated by an allosteric pathway. Expanding these approaches with the use of piezo droplet injectors simplifies reaction initiation for systems that are not naturally amenable to light activation. The "Liquid Application Method for time-resolved Analysis" (LAMA) method is very efficient using only minimal amounts of protein (1-3 mg) and ligand (1.5-3 $\mu \mathrm{l})$ per time point. In combination with the HARE approach and Glucose Isomerase as a model system, we were able to observe substrate binding and an open ring conformation of glucose during its catalytic cycle. These results demonstrate the relevance and applicability of time-resolved approaches to provide insights into the functional dynamics of proteins.

Acta Cryst. (2020). A76, a51 